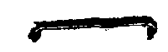

NASA Technical Memorandum 102056

\title{
Holographic Interferometry With an Injection Seeded Nd:YAG Laser and Two Reference Beams
}

Arthur J. Decker

Lewis Research Center

Cleveland, Ohio

(NASA-TH-102056) HOL OGRAPHIC INTERFEBCAETRY

189-24591

UITH AN IUJECTION SEEDED NA:YAG LASEB AND

$T$ TO REFEREXCE BEAHS (NASA. Levis Research

Center) $23 \mathrm{p}$

CSCL $20 \mathrm{~F}$

Unclas

$63 / 35$

0211713

May 1989

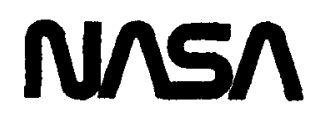




\title{
HOLOGRAPHIC INTERFEROMETRY WITH AN INJECTION SEEDED Nd:YAG LASER AND TWO REFERENCE BEAMS
}

\author{
Arthur J. Decker \\ National Aeronautics and Space Administration \\ Lewis Research Center \\ Cleveland, Ohio 44135
}

\begin{abstract}
The performance of twin injection seeded Nd:YAG lasers is compared with the performance of an argon-ion laser for recording dual-reference-beam holograms in AGFA 8E56 emulsion. Optical heterodyning is used to measure interference, and the results are expressed in terms of heterodyning signal level and intensity signal-to-noise. The Nd:YAG laser system is to be used for optical inspections of structures for cracks, defects, gas leaks, and structural changes.
\end{abstract}




\section{INTRODUCTION}

The injection seeded Nd:YAG technology increases the efficiency of routine optical inspections using dual-reference-beam holographic interferometry. This paper will compare the performances of argon-ion and Nd:YAG lasers for recording dual-reference-beam holograms in Agfa 8 E56 emulsion.

The literature contains many discussions of phase-sensitive electronic interferometry and electronic moire. Dändliker and colleagues have done pioneering work with the holographic implementation. ${ }^{1-3}$ Ruby-laser, dual-reference-beam, holographic interferometry has been discussed by Swain and Tansey, and Wagner has described a version using Nd:YAG. Lewis Research Center has ongoing projects to use the phase sensitive methods for measurements of flow properties ${ }^{6,7}$ and more recently for structural inspections.

An injection-seeded Nd:YAG laser and dual-reference-beam holographic interferometry have been used at Lewis Research Center for crack detection in a composite subjected to high cycle fatigue. Figure 1 compares crack-detection inspections of a sample of composite material performed with argon-ion and injection-seeded Nd:YAG lasers. Both plots show numerically evaluated second derivatives of the raw interference phase measured from fringe patterns recorded by dual-reference-beam, double-exposure, holographic interferometry. 
Optical heterodyning was used to perform the measurements. A point load was applied to the sample between exposures of the argon-ion laser hologram. The Nd:YAG hologram was a double exposure of the sample, recorded while the sample was vibrating in its first cantilever mode. The second derivatives are seen to deviate significantly from linear in the neighborhood of the crack. The argon-ion and Nd:YAG holograms were equally effective for crack detection.

Nd:YAG lasers offer several advantages over argon-ion and ruby lasers for performing routine optical inspections such as the crack detection example. The lasers radiate from 5 to $50 \mathrm{Q}$-switched pulses per second, making it easy to align the beams relative to an object. The second harmonic of the Nd:YAG laser at $532 \mathrm{~nm}$ is close to the wavelength of argon-ion at $514.5 \mathrm{~nm}$, so that two argon-ion beams can be used to reconstruct the fringe patterns for measurement with minimum wavelength-shift error. The high repetition rates of the lasers make it possible to perform many optical inspections of short-lived structures. Dual-reference-beam, holographic interferometry can be performed with holographic emulsions on film ${ }^{7}$ when it is necessary to record more than one hologram per second.

These benefits should be weighed with caution. There are relatively few examples of Q-switched, Nd:YAG holography, ${ }^{5,8-10}$ although Nd:YAG itself has a good commercial track record. Beam smoothing methods such as spatial filtering are fairly difficult to perform with Nd:YAG as with 
ruby lasers.

A startling property of Nd:YAG laser holography is the change in performance of the common silver halide emulsions used for holography. Pulsed laser holograms, with ruby in mind, are quoted as having a factor of 2 to 4 reciprocity failure. ${ }^{11}$ It is shown in this paper that the short pulses from the Nd:YAG laser ( $5 \mathrm{nsec}$ to $15 \mathrm{nsec}$ ) result in a factor of 4 to 6 reciprocity failure. Everything else equal, this means that a Nd:YAG laser will require 4 to 6 times the energy of an argon-ion exposure for a given hologram situation. This disturbing property is ameliorated significantly by two facts. First, heterodyne holographic interferometry is adequate for optical inspections even though the detected signals vary by 2 to 3 orders of magnitude. The factors that determine sensitivity are not affected very much by the holographic technique, consequently big mistakes are forgiven. The second fact, substantiated by the measurements reported herein, is that increasing the temperature of the developer by 6 to $10^{\circ} \mathrm{F}$ improves the performance of the silver-halide emulsions substantially, when they are exposed to the second harmonic of a Q-switched, Nd:YAG laser.

In this paper, argon-ion at $514.5 \mathrm{~nm}$ is compared with Nd:YAG at 532 $\mathrm{nm}$ for the Agfa 8E56 emulsion. Hardware, experimental setup, and results are discussed. The trends established appear to be similar for other holographic emulsions such as Agfa 10E56 and Kodak SO 253. 


\section{INSTRUMENTATION}

The principal hardware for this work was a pair of Quantel International YG580 Series oscillator, amplifier, frequency-doubler combinations and a single injection seeder. The three lasers were assembled by the manufacturer and mounted on a single, covered, vibration-isolation table.

The injection seeder causes the $\mathrm{Q}-$ switched oscillators to tend to operate in a single longitudinal mode. ${ }^{12}$ In the absence of the injection seeder, Q-switched pulses build up from spontaneous emission propagating within the oscillator spatial mode. The large line-width of the spontaneous emission and the moderately high gain of Nd:YAG assure that many longitudinal modes lase. Consequently, the coherence length of the laser is very short (less than $1 \mathrm{~cm}$ ), making it difficult to record holograms of objects which have any surface relief. During injection seeding, the output of the injection seeder is mode matched using telescopes to the spatial modes of the Q-switched oscillators. The injection-seeder light is directed into the oscillators by reflecting it off the $\mathrm{Q}-\mathrm{switch}$ polarizers. The three lasers are tuned to the same frequency using temperature tuning and by using piezoelectric control of the positions of the high reflectors of the oscillators. When this package is operating correctly, the Q-switched pulses build up much earlier than would happen if the spontaneous emission were in control, and the laser will operate in a single longitudinal mode. The injection-seeder package is able to monitor this build-up time 
and to minimize the build-up time by adjusting the piezoelectric controlled positions of the high reflectors.

The two lasers each produce about $500 \mathrm{~mJ}$ per pulse of $532 \mathrm{~nm}$ light at 10 pulses per second. The full width at half maximum power of a pulse is about 7 nanoseconds. The time separation between the two laser pulses can be adjusted from about 1 to 1000 microseconds for double exposure holograms. The spectroscopic line-width of a laser pulse is about .005 $\mathrm{cm}^{-1}$ as confirmed by a Michelson interferometer and holography. The $1 / \mathrm{e}^{2}$ diameter of a green laser beam is about $8 \mathrm{~mm}$. Roughly speaking, these properties translate into an ability to record good dual-reference-beam holograms (one laser for each reference beam) of a diffusely reflecting object with a surface relief of about $0.5 \mathrm{~m}$ and a maximum projected dimension of about $1.0 \mathrm{~m}$.

There are some cautions in buying and using such a system in addition to proper use of the holographic emulsions. One tacit assumption is that the two lasers produce beams which are identical geometrically, have the same frequency, and are precisely coaxial at the object. Otherwise systematic phase errors and relative speckle-pattern shifts can occur. Differences in the beam properties have not so far affected the optical inspection applications. Beam differences and misalignment could become important if more exact numerical results are required. A more noticeable effect has been less reliability in getting an injection-seeded second pulse as the pulse separation decreases, especially below 100 microseconds. 
A second principal item is a Coherent Innova 90 argon--ion laser used for reconstruction and for fringe measurements. This laser was also used to record the argon-ion holograms which were compared with the Nd:YAG holograms. For reconstructions and measurements, the laser was always operated at a wavelength of $514.5 \mathrm{~nm}$, at a power of $325 \mathrm{~mW}$, and with its etalon installed. The $1 / \mathrm{e}^{2}$ diameter of this laser beam is about $1.5 \mathrm{~mm}$.

The argon-ion laser and the fringe measurment station are supported by a large vibration-isolation table. Fringe measurements are accomplished with a setup similar to that described by Dändliker. ${ }^{1}$ A dual-input, lock-in amplifier is used as a phase sensitive detector in place of a zero-crossing meter. The light detectors are fiber bundle, photomultiplier combinations. Pre-amplification and pre-filtering are available. A precision xyz positioning stage moves a detector over an area $30 \mathrm{~cm}$ by $30 \mathrm{~cm}$ in micrometer increments. The positioning stage has been checked against a laser interferometer, and systematic differences are within 10 micrometers over the central $25 \mathrm{~cm}$ by $25 \mathrm{~cm}$ of travel. The entire measurement process is automated under the control of an IBM AT system with commercial and locally generated software. The performance of the system is comparable to published values. ${ }^{1-3}$ Three standard deviations of scatter in measured phase range from $1 / 200$ to $1 / 300$ fringe-shift when the reference-beam interangle exceeds the angular diameter of the scene and from $1 / 60$ to $1 / 100$ fringe-shift when a small reference-beam interangle is employed. A small reference interangle of a 
few hundredths of an angular degree is required, if holograms are recorded in one location at one wavelength and transported for reconstruction at a different wavelength in a different location. The small reference-beam interangle makes the whole process relatively insensitive to misalignment and fairly easy to set up. The small beam interangle was used to record, reconstruct, and measure all data reported in this article.

It is much more difficult to feel confidence in measurements of intensity than in measurements of phase. This work required measurements of exposures and beam irradiance ratios. A Newport model 820 laser power meter was used to measure the argon-ion values. The measurements and corresponding performance of the Agfa 8E56 emulsion agree well with published values ${ }^{13}$. The corresponding pulsed laser measurements are less creditable. A Scientech 362 power energy meter yielded the most consistent agreement between input energy and measured exposure values. Pulsed-laser beam-irradiance ratios were measured through a diffuser in the hologram plane using photodiode, neutral-density-filter combinations and energy-meter, neutral-density-filter combinations. Combinations of neutral density filters were used to prevent saturation of the photodiode and to achieve roughly equal detector outputs for the object and reference beams. The beam ratio was then computed from the filter values and the detector readings in the plane of the hologram. The pulsed-laser exposures are estimated to be accurate to 10 percent. Beam ratio measurements, accomplished with photodiodes and power meters, differ by 
as much as 30 percent.

The experiment and results which use the above instrumentation to compare the performances of AGFA $8 E 56$ emulsion for argon-ion and Nd:YAG dual-reference-beam holographic interferometry are summarized in the next section. 


\section{EXPERIMENT AND RESULTS}

The argon-ion and Nd:YAG holograms were recorded of the same object using the same recording geometry and the same hologram handling procedures. The object was a rectangular blade of aluminum alloy, painted flat white, and mounted in a vise. The rectangle extending above the vise was $87 \mathrm{~mm}$ by $58 \mathrm{~mm}$. The object surface was parallel to and centered on the 4 " by 5 " glass holographic plates. The object distance from the emulsion was $50 \mathrm{sm}$. The laser beams were parallel to the supporting table. The central ray of the reference beam was at 45 degrees relative to the normal to the plate. The diverging lens for the reference beam was $500 \mathrm{~mm}$ distant from the center of the holographic plate. The object-beam and reference-beam path lengths were adjusted to be equal at the center of the glass plate. All exposure measurements were made at the center of the glass plate. The reference-beam interangle was about $1.0 \mathrm{mrad}$ for the argon-ion laser and about $0.5 \mathrm{mrad}$ for the Nd:YAG laser.

The beam irradiances for the argon-ion holograms were measured with the Newport model 820 laser power meter. The detector was pointed at the object and perpendicular to the emulsion for all measurements. Variable density beam splitters were used to vary the beam irradiance ratios. Exposure time multiplied by irradiance determined the total exposure. 
The exposures for the Nd:YAG holograms were determined less directly as stated in the previous section. The Scientech meter was located at the hologram position and pointed at the reference source. The energy per pulse was divided by the detector area and multiplied by the cosine of the angle of incidence to determine the reference-beam contribution to the exposure. These irradiances agreed well with irradiances computed from an assumed Gaussian profile, using a $1 / \mathrm{e}^{2}$ diameter equal to the front-burn diameter on light-sensitive chart paper, the focal length of the diverging lens, and the measured energy at the lens. The object-beam contribution to the exposure was calculated using the measured beam ratio. The exposures and beam ratios were varied by changing the powers of the object and reference-beam diverging lenses and by varying the distance between the object and the object-illumination lens.

The fringe patterns for the argon-ion holograms were created by bending the blade slightly between exposures. A precision actuator displaced the blade tip $2 \mu \mathrm{m}$ between exposures.

The fringe patterns for the Nd:YAG holograms were created by recording double-exposure holograms of the vibrating blade. The blade was driven in its first bending mode using a power-oscillator, speaker combination. The speaker drive and time separation between exposures were adjusted to produce about the same number of fringes as for the argon-ion holograms.

The holograms were processed in the same way except for developer 
temperature. A fairly standard development and bleaching procedure was used. Chemicals were changed frequently, usually after 4 holograms. The holograms were developed with continuous hand agitation, 4 at a time, for 4 min. in Kodak D-19. Development was followed by $30 \mathrm{sec}$. rinse in running water at $68^{\circ} \mathrm{F}$. The holograms were then fixed in Kodak Rapid Fix with Hardener for 4 min. with continuous hand agitation. The holograms were then rinsed for $3 \mathrm{~min}$. in $68^{\circ} \mathrm{F}$ running water. The holograms were then bleached until clear using a potassium ferricyanide, potassium bromide bleach (35 $\mathrm{g}$ potassium ferricyanide, $10 \mathrm{~g}$ potassium bromide in $1000 \mathrm{ml}$ of water). The bleached holograms were rinsed for 3 min. in running water and dried with an air drier.

All measurements were made immediately after processing, although they can be made after weeks of storage. Two kinds of measurements were used to characterize the performance of a hologram: heterodyne signal level and visual signal-to-noise. The reconstructed virtual image and fringes were re-imaged at unity magnification for both kinds of measurements.

The heterodyne signal level is proportional to the magnitude of the cross interference term in the time varying fringe pattern. The fringe patterns, localized on the blade image, were imaged with a $\mathrm{f} / 5.6$ lens on a $1.5 \mathrm{~mm}$ diameter fiber bundle for the heterodyne measurements. For each hologram, the computer controlled measurement system acquired 150 measurements of signal level over a $30 \mathrm{~mm}$ by $30 \mathrm{~mm}$ patch of the fringe 
pattern. The statistical mode of the signal level was estimated from a contour plot of the signal. The signal level varied by as much as 2 to 1 for the argon-ion holograms and as much as 10 to 1 for the Nd:YAG holograms. The actual intended application of dual-reference-beam holographic interferometry is to measure interference phase. The major source of error in measuring phase is overlapping cross reconstructions. ${ }^{1}$ Our experience is that large variations in signal level have little effect on the application to optical inspection.

A visual signal-to-noise of the reconstructed images was also defined and measured. This quantity was measured with the Newport power meter. The signal-to-noise is the power measured in the brightest part of the $30 \mathrm{~mm}$ by $30 \mathrm{~mm}$ patch divided by the background power measured always at the same point away from the blade. The holograms were recorded with a black cloth in the background to avoid contributions from other objects. The measurements were made with both reconstruction beams turned on and with one bearn frequency shifted to time-average the fringe pattern. The reconstructed image actually consists of two direct reconstructions and two cross reconstructions.

The results are summarized by Figs. 2 and 3. Figure 2 shows a figure of merit for dual-reference-beam holographic interferometry for the argon-ion and injection-seeded Nd:YAG lasers. The figure of merit is defined to be the product of the relative heterodyne signal level and the visual signal-to-noise measurement. A figure of merit permits an 
estimate of the tradeoff between the increase in signal level at lower beam ratios and the accompanying increase in nonlinear effects. The figure of merit is plotted against beam irradiance ratio. Figure 3 is a plot of the visual signal to noise against beam irradiance ratio.

The results shown for the argon-ion holograms represent a single developer temperature of $68^{\circ} \mathrm{F}$ and measured exposures within 10 percent of $33 \mu \mathrm{J} / \mathrm{cm}^{2}$. The results shown for the injection-seeded Nd:YAG laser represent developer temperatures of $68^{\circ} \mathrm{F}, 74^{\circ} \mathrm{F}, 76^{\circ} \mathrm{F}$, and $78^{\circ} \mathrm{F}$. The measured exposures for Nd:YAG range from 120 to 130 $\mu \mathrm{J} / \mathrm{cm}^{2}$. 


\section{DISCLSSION}

The argon-ion, 8E56 performance shown in Fig. 2 is a good choice for comparison with the accompanying Nd:YAG, 8E56. The exposure is exactly in the range recommended to achieve an optical density of unity ${ }^{13}$, which is desirable for bleached holograms. This exposure is also desirable for dual-reference-beam holograms. Exposures 50 percent higher and 50 percent lower resulted in significantly lower figures of merit. The maximum heterodyne signal for the argon-ion holograms was actually measured for the heavier exposure, but the signal-to-noise was more than proportionately reduced.

A developer temperature of $68^{\circ} \mathrm{F}$ produces a comparatively poor performance for Nd:YAG, 8E56 as shown in Figs. 2 and 3, even though the interference phase is easy to measure. The performance improves significantly for warmer developer. In fact, the signal-to-noise in Fig. 3 does not differ much from the argon-ion values when the developer temperatures are $74^{\circ} \mathrm{F}$ and higher. The Nd:YAG exposures are about 4 times the argon-ion exposures used for the comparison holograms. Even better performance was obtained with heavier exposures at constant beam irradiance ratios. The reciprocity failure appears to range from 4 to 6 .

Lewis Research Center has used other emulsions for Nd:YAG laser holography including Kodak SO 253 and Agfa 10E56.10 These two emulsions performed similarly when used for flow visualization 


\begin{abstract}
applications. 10 The other emulsions show the same trends exhibited for Agfa 8E56. Heavier exposures and higher developer temperatures are required.
\end{abstract}




\section{CONCLUDING REMARKS}

A laser system consisting of two injection seeded Nd:YAG lasers has been used with the Agfa $8 \mathrm{E} 56$ emulsion on glass plates to record dual-reference-beam holograms, where the resultant fringe patterns were detected and measured by optical heterodyning. A practical, although not optimum, holographic technique is to increase the exposure level 4 times above the optimum argon-ion exposure for the same setup and to increase the temperature of the Kodak D-19 developer to $76^{\circ} \mathrm{F}$. A beam-irradiance ratio of about 10 to 1 is desirable, although higher ratios produce measurable results.

The laser system must then produce at least 4 times the energy that would be estimated from argon-ion holography for a given setup involving a particular diffusely reflecting object and a given beam ratio. However, the electronic detection methods allow fringe patterns to be measured from holograms recorded with much lower exposures and much higher beam ratios. Roughly speaking, $1 \mathrm{~J}$ total of $532 \mathrm{~nm}$ light allows dual-reference-beam, Fresnel holograms to be recorded usefully of flat-white painted objects with maximum projected dimensions of $1 \mathrm{~m}$. This statement applies to the Agfa 8E56 emulsion. Similar detailed studies of other recording materials would be useful. The Kodak SO 253 emulsion would be a good candidate, since that emulsion on film can be used with a Nd:YAG laser to record many holograms and to perform 
many optical inspections of short-lived objects.

The use of the Nd:YAG, Agfa $8 E 56$ combination for recording display holograms is a little more questionable. The desired exposure level is probably 6 times that of argon-ion. Actually, the holograms recorded with lower exposures and proper development are good: they suffer only when compared with CW laser holograms. Commercial ruby lasers with their larger coherence lengths, higher energies, and lesser reciprocity failure are a significant competitor for recording display holograms and holograms of very large objects. The dual-beam, injection-seeded, Nd:YAG laser is most useful for efficient performance of the optical inspection applications discussed in the first section because of its high pulse repetition rate and because its second harmonic has a wavelength close to the wavelengths produced by the argon-ion laser.

The author acknowledges the invaluable assistance of Kenneth $\mathrm{E}$ Weiland of the Technical Services Directorate of Lewis Research Center in recording and processing the holograms used for this study. 


\section{REFERENCES}

1. R. Dändliker, "Heterodyne Holographic Interferometry," in Progress in Optics, Vol. 17, E. Wolf, Ed., (North Holland, New York, 1980), pp. 1-84.

2. R. Dändliker, D. Eliasson, D. Ineichen, and F.M. Mottier, "Quantitative Determination of Bending and Torsion Through Holographic Interferometry," in The Engineering Uses of Coherent Optics, E.R. Robertson, Ed., (Cambridge University Press, Cambridge, 1976), pp. 99-117.

3. R. Dändliker and R. Thalmann, "Heterodyne and Quasi-Heterodyne Holographic Interferometry," Opt. Eng. 24, 824 (1985).

4. D. Swain and R. Tansey, "Heterodyne Holographic Interferometry," in Automated Reduction of Data from Images and Holograms, NASA CP-2477, G. Lee, J.D. Trolinger, and Y.H. Yu, Eds., (National Aeronautics and Space Administration, Washington, D.C., 1985), pp. $249-260$.

5. J.W. Wagner, "Triple-Exposure Dual-Reference Holographic Recording for Heterodyne Analysis of Transient Phenomena," Appl. Opt. 24, 2937 (1985).

6. A.J. Decker and J. Stricker, "A Comparison of Electronic Heterodyne Moire Deflectometry and Electronic Heterodyne Holographic Interferometry for Flow Measurements," SAE Paper 851896 (Society of Automotive Engineers, Warrendale, PA, 1985). (NASA TM-87071). 
7. A.J. Decker, "Beam-Modulation Methods in Quantitative and Flow Visualization Holographic Interferometry,', in Advanced Instrumentation for Aero Engine Components, AGARD-CP-399 (Advisory Group for Aerospace Research and Development, NeuillySur-Seine, France, 1986), pp. 34-1 to 34-16. (NASA TM-87306).

8. F.C. Way, "The Pulsed Nd:YAG Holographic Laser-Present Status and Applications," in Electro-Optical Systems Design Conference and International Laser Exposition, Proceedings of the Technical Program, (Industrial and Scientific Conference Management, Inc., Chicago, 1975), pp. 760-766.

9. A.J. Decker, “Holographic Cinematography of Time-Varying Reflecting and Time-Varying Phase Objects Using a Nd:YAG Laser,", Opt. Lett. 7, 122 (1982).

10. A.J. Decker, "Evaluation of Diffuse-Illumination Holographic Cinematography in a Flutter Cascade,' NASA TP-2593 (1986).

11. R.J. Collier, C.B. Burckhardt, and L.H. Lin, in Optical Holography, (Academic Press, New York, 1971), pp. 311-336.

12. Y.K. Park, G. Giuliani, and R.L. Byer, "Stable Single-Axial-Mode Operation of an Unstable-Resonator Nd:YAG Oscillator by Injection Locking,', Opt. Lett. 5, 96 (1980).

13. J.W. Gladden and R.D. Leighty, "Recording Media," in Handbook of Optical Holography, H.J. Caulfield, ed., (Academic Press, New York, 1979), pp. 277-298. 


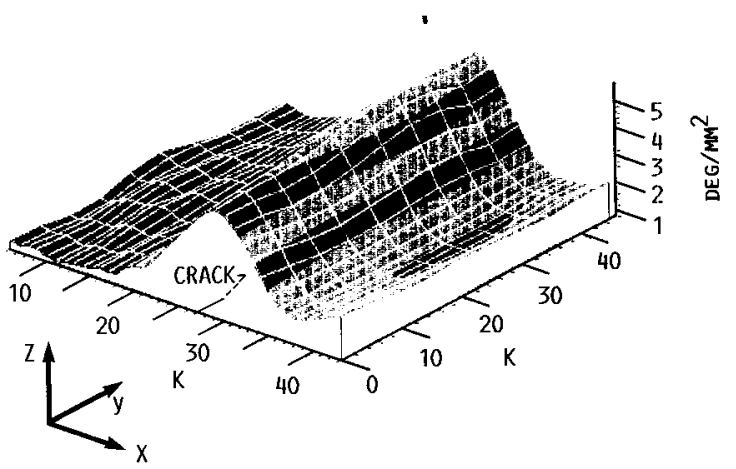

(a) ARGON-ION INSPECTION.

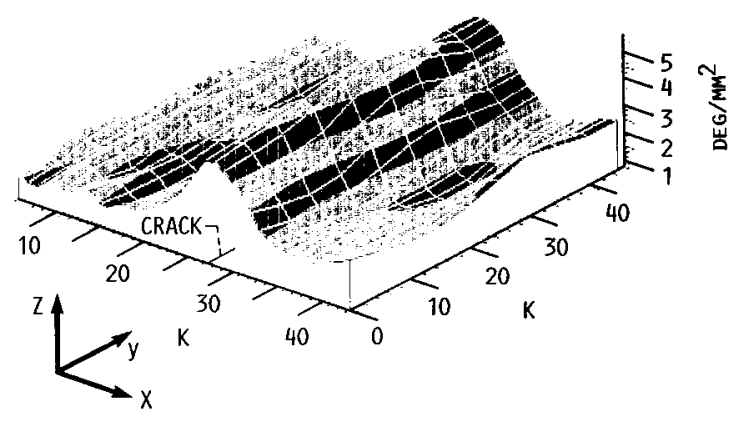

(b) Nd:YAG INSPECTION

FIGURE 1. - CRACK DETECTION FROM SECOND DERIVATIVES OF RAW INTERFERENCE PHASE MEASURED BY OPTICAL HETERODYNING OF DOUBLE-EXPOSURE, DUAL-REFERENCE-BEAM HOLOGRAMS OF SAMPLE IN BENDING. SECOND DERIVATIVES ARE ESTIMATED WITH RESPECT TO $X$ USING CENTRAL DIFFERENCES AND MEASUREMENTS SEPARATED BY $3 \mathrm{~mm}$.

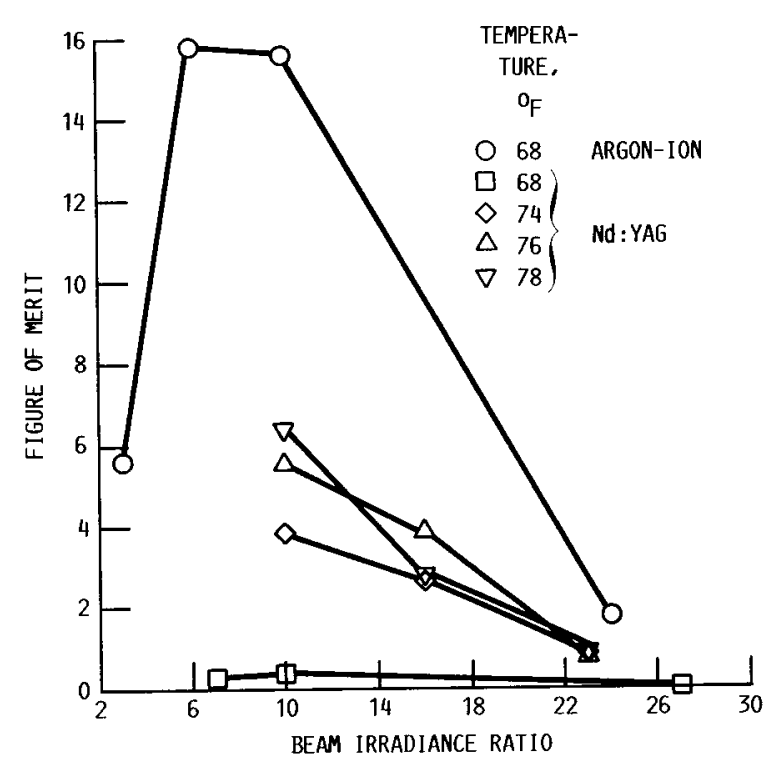

FIGURE 2. - FIGURES OF MERIT (PRODUCT OF HETERODYNE SIGNAL LEVEL AND INTENSITY SIGNAL-TO-NOISE) FOR ARGON-ION AND Nd:YAG DUAL-REFERENCE-BEAM HOLOGRAMS IN Agfa 8E56 AS A FUNCTION OF BEAM IRRADIANCE RATIO. THE ARGON-ION HOLOGRAMS WERE EXPOSED AT ABOUT $33 \mu \mathrm{J} / \mathrm{cm}^{2}$ AND DEVELOPED AT $68^{\circ} \mathrm{F}$. THE Nd:YAG HOLOGRAMS WERE EXPOSED AT ABOUT $125 \mu \mathrm{J} / \mathrm{cm}^{2}$ AND DEVELOPED FROM 68 TO $78^{\circ} \mathrm{F}$.

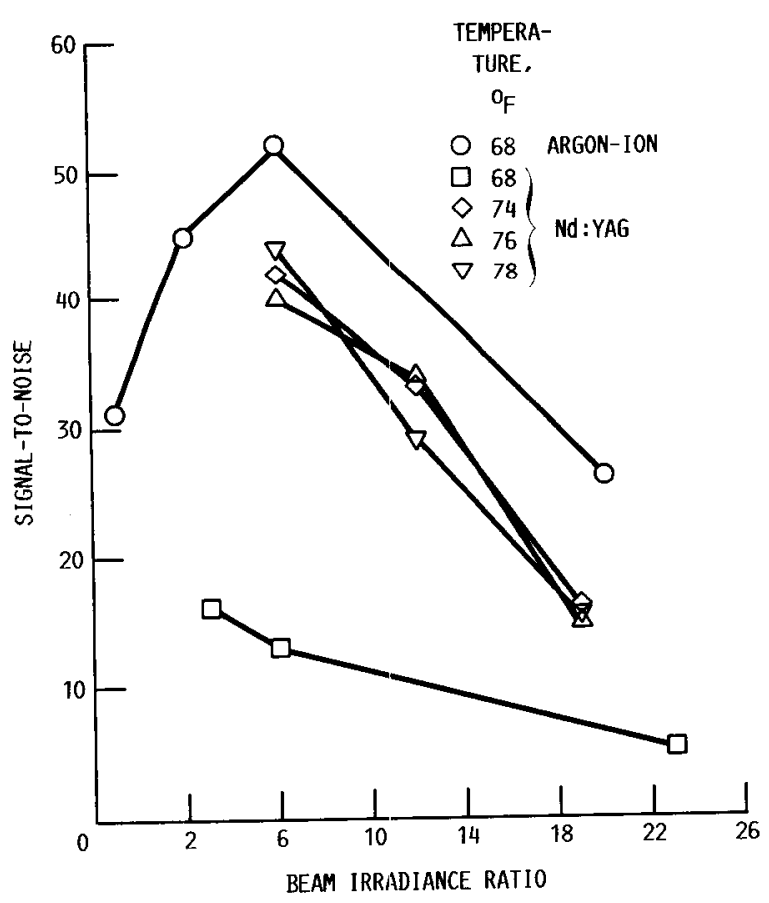

FIGURE 3. - INTENSITY SIGNALS-TO-NOISE FOR ARGON-ION AND Nd : YAG DUAL-REFFERENCE-BEAM HOLOGRAMS AS FUNCTION OF BEAM IRRADIANCE RATIO. EXPOSURES AND DEVELOPMENT TIMES ARE THE SAME AS IN FIGURE 2. 


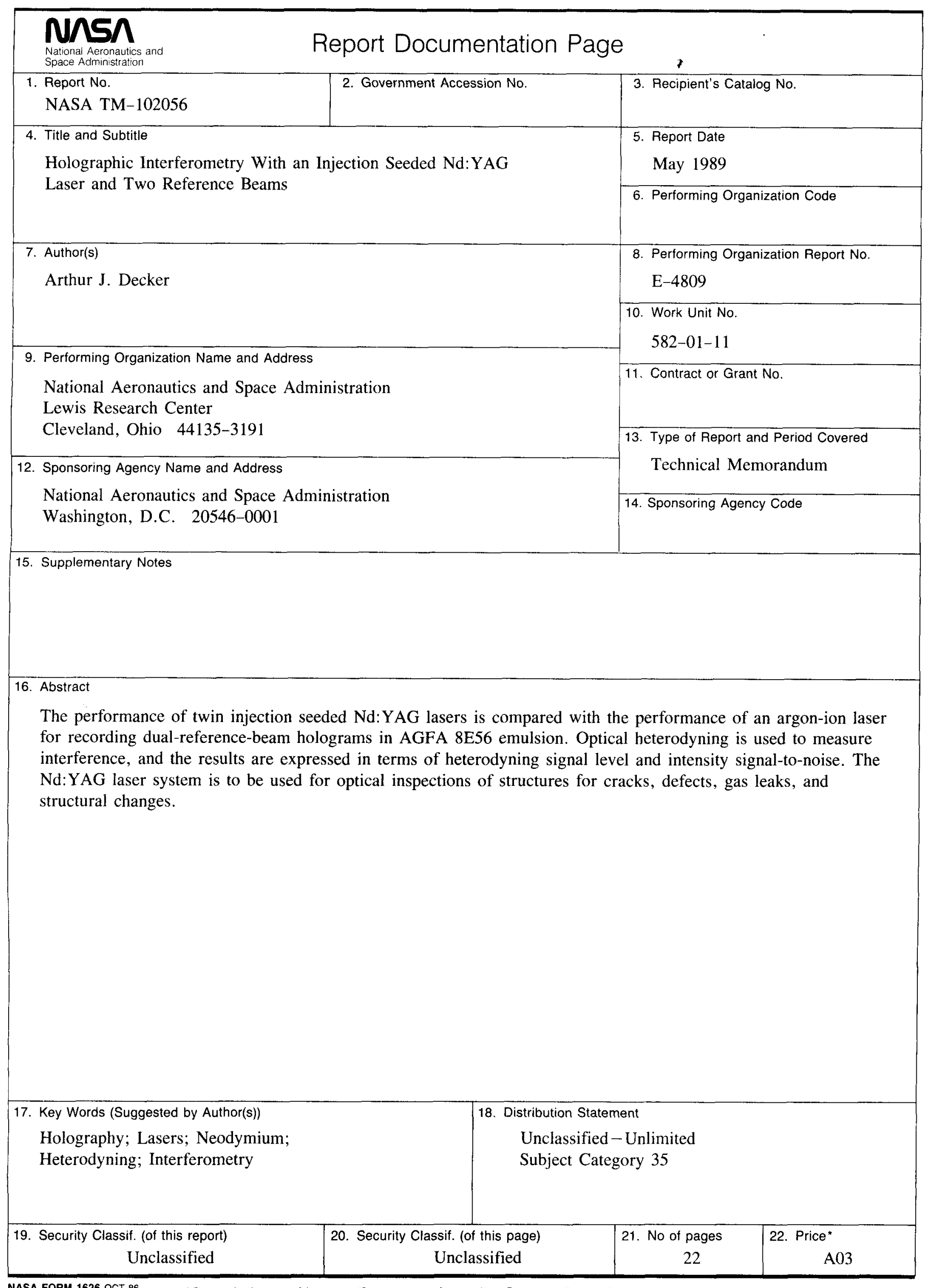

